\title{
Dispersion of metal-insulator-metal plasmon polaritons probed by cathodoluminescence imaging spectroscopy
}

\author{
Martin Kuttge, ${ }^{1, *}$ Wei Cai ${ }^{2,3}$ F. Javier García de Abajo, ${ }^{2}$ and Albert Polman ${ }^{4}$ \\ ${ }^{1}$ Center for Nanophotonics, FOM-Institute AMOLF, Sciencepark 113, 1098 XG Amsterdam, The Netherlands \\ ${ }^{2}$ Instituto de Óptica-CSIC, Serrano 121, 28006 Madrid, Spain \\ ${ }^{3}$ The Key Laboratory of Weak-Light Nonlinear Photonics, Ministry of Education and TEDA Applied Physics School, \\ Nankai University, Tianjin 300457, China \\ ${ }^{4}$ Center for Nanophotonics, FOM-Institute AMOLF, Kruislaan 407, 1098 SJ Amsterdam, The Netherlands
}

(Received 5 June 2009; revised manuscript received 6 July 2009; published 24 July 2009)

\begin{abstract}
Cathodoluminescence imaging spectroscopy is used to excite and characterize the resonant modes of FabryPerot resonators for surface plasmon polaritons confined in a metal-insulator-metal (MIM) geometry. The smallest MIM plasmon wavelength derived from the observed mode pattern is found to be $160 \mathrm{~nm}$ in cavities with a $10 \mathrm{~nm} \mathrm{SiO} \mathrm{S}_{2}$ layer for a free-space wavelength of $645 \mathrm{~nm}$. The measured wavelength agrees well with values from analytical dispersion relation calculations. Calculations of the excitation probability show that the resonant excitation of MIM plasmons depends strongly on the electron energy due to phase retardation effects resulting from the finite electron velocity.
\end{abstract}

DOI: 10.1103/PhysRevB.80.033409

PACS number(s): 73.20.Mf, 41.60.- m, 78.60.Hk

Surface plasmon polaritons (SPPs) are electromagnetic waves that propagate at the surface of a metal. Their evanescent field tail typically extends several hundreds of nanometers into the surrounding dielectric. Near the surface plasmon frequency, the SPP dispersion relation deviates strongly from the light line toward larger wave vectors, thus enabling short plasmon wavelengths at optical frequencies.

The dispersion relation of plasmons can be further tuned in metal-insulator-metal (MIM) geometries, in which the plasmon field is confined in a $10-100 \mathrm{~nm}$ dielectric gap between two metal layers. ${ }^{1}$ Due to intercoupling of plasmons at both metal-dielectric interfaces, plasmon modes with symmetric and antisymmetric magnetic field distribution exist, of which the latter shows the largest dispersion and highest loss. ${ }^{2,3}$

The unique properties of MIM plasmons are currently a research topic that attracts interest. The propagation of MIM modes was measured in slot waveguides ${ }^{4}$ and the dispersion relation was determined using near-field microscopy. ${ }^{5}$ Twodimensional negative refraction of plasmons was demonstrated in the visible, ${ }^{6}$ and also confirmed theoretically. ${ }^{7}$ In these studies, MIM plasmons were excited by an external light source using in-coupling through slits in one of the metal cladding layers.

In this paper, we present cathodoluminescence (CL) spectroscopy measurements of MIM plasmon modes. We use the electron beam of a scanning electron microscope (SEM) that traverses through the layer stack to directly excite MIM plasmons confined in Fabry-Perot resonator structures. Measurements of the mode structure allow us to determine the MIM plasmon wave vector. A plasmon wavelength as small as 160 $\mathrm{nm}$ was found for a $10 \mathrm{~nm} \mathrm{SiO}$ layer. The observed wave vectors agree well with analytical calculations of the dispersion relation. Calculations of the excitation efficiency of MIM plasmons by an electron beam show the possibility of selective mode excitation.

The MIM samples were prepared using physical vapor deposition from a thermal evaporation source onto a cleaned silicon substrate. The layer stack consisted of subsequent layers of $10 \mathrm{~nm}$ chromium, $100 \mathrm{~nm}$ silver, 10,30 , or $50 \mathrm{~nm}$ $\mathrm{SiO}_{2}, 100 \mathrm{~nm}$ silver, and $10 \mathrm{~nm}$ chromium. A control sample was evaporated at the same time and shielded by a shutter during the $\mathrm{SiO}_{2}$ evaporation leaving it without the $\mathrm{SiO}_{2}$ layer.

The Cr layers were deposited to damp out single-interface SPPs propagating at the surface. Figure 1(c) shows the propagation length as a function of wavelength for the sym-

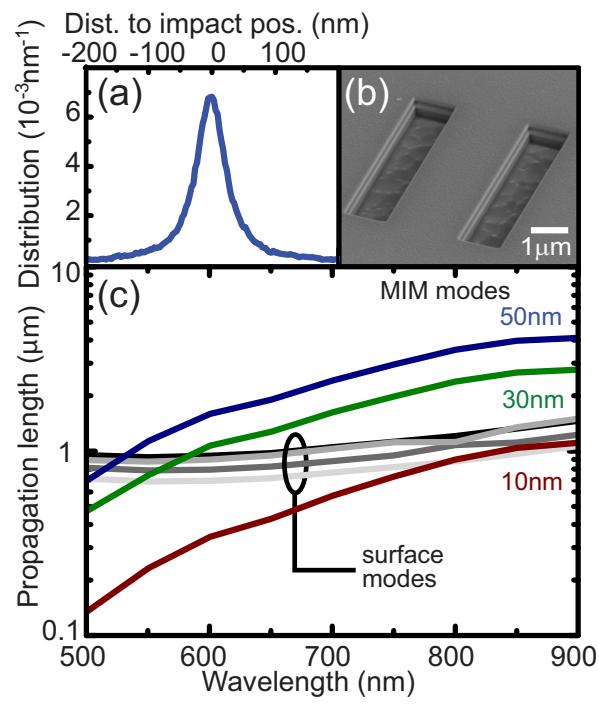

FIG. 1. (Color online) (a) Electron distribution as a function of position for $30 \mathrm{keV}$ electrons in the center of the $\mathrm{SiO}_{2}$ layer of the MIM structure along a direction perpendicular to the impinging beam. The electron distribution was calculated using a Monte-Carlo simulation. (b) SEM image of a one-dimensional Fabry-Perot resonator in a MIM structure. (c) Calculated propagation length as a function of wavelength for MIM and surface SPP modes for samples with 10,30 , or $50 \mathrm{~nm} \mathrm{SiO}_{2}$ layer and a silica-free control sample. The propagation lengths were calculated using a vectorial finite-difference mode solver. 
metric MIM modes in comparison to the SPP mode propagating on the top interface, calculated using a vectorial finite-difference mode solver included in a commercialsimulation package. ${ }^{8}$ The propagation length for the top SPP modes varies only slightly depending on the underlying layer stack. For the longest wavelengths under consideration it is a factor of 3 lower than the propagation length for the MIM modes inside the 30 and $50 \mathrm{~nm} \mathrm{SiO}_{2}$ layers. Only for the 10 $\mathrm{nm} \mathrm{SiO}_{2}$ layer the propagation length of the Ag-surface mode exceeds the propagation length of the MIM mode. Note, that the asymmetric MIM mode, being in cut-off for the studied $\mathrm{SiO}_{2}$ layer thicknesses, has 2-3 orders of magnitude shorter propagation lengths and will therefore not be observed.

The $30 \mathrm{keV} \mathrm{Ga}{ }^{+}$beam of a focused ion-beam system was used to structure resonator structures into the MIM stack. The focused ion beam (beam current $48 \mathrm{nA}$ ) etched through the entire layer stack and approximately $200 \mathrm{~nm}$ into the underlying silicon substrate. Figure 1(b) shows an SEM image of a Fabry-Perot resonator structured into a layer stack with a $50 \mathrm{~nm} \mathrm{SiO}_{2}$ layer imaged under an angle of $52^{\circ}$ off the surface normal. The one-dimensional cavity is formed by two parallel grooves of $1000 \mathrm{~nm}$ width that are spaced by a distance of $2000 \mathrm{~nm}$. The groove length is $5000 \mathrm{~nm}$. The Si substrate, the metal layers, and the $\mathrm{SiO}_{2}$ can be easily identified. The contrast between $\mathrm{Ag}$ and $\mathrm{Cr}$ is too small to be seen in the image.

We use spatially resolved cathodoluminescence imaging spectroscopy ${ }^{9}$ to excite plasmon modes in the MIM structures and measure the emission in the far field. The samples are excited by the $30 \mathrm{keV}$ electron beam of a SEM, which is focused to a $10 \mathrm{~nm}$ spot onto the sample surface. Due to electron scattering in the upper metal layers the beam diameter is increased as it penetrates into the layer stack. Figure 1(a) shows the result of a Monte-Carlo simulation ${ }^{10}$ of the electron beam profile at the center of the $\mathrm{SiO}_{2}$ layer, assuming a $10 \mathrm{~nm}$ diameter incident beam. The beam diameter is increased to approximately $50 \mathrm{~nm}$. A parabolic mirror (acceptance angle $1.4 \pi s r$ ) placed above the sample collects the emitted light and guides it to a spectrometer, in which the light is spectrally resolved and detected using a liquidnitrogen cooled charge-coupled device array. The collected spectra are corrected for system response by measuring the transition radiation spectrum for a known gold sample and normalizing it to the calculated transition radiation spectrum. ${ }^{9}$

Figure 2 shows the CL emission as a function of wavelength and electron-beam position for a line scan across three Fabry-Perot resonator structures: (a) the control sample without $\mathrm{SiO}_{2}$ layer, (b) the MIM stack with a $50 \mathrm{~nm} \mathrm{SiO}$ layer, and (c) the stack with a $30 \mathrm{~nm} \mathrm{SiO}_{2}$ layer. In all three scans the grooves are clearly resolved at the top and bottom of the image as areas of low emission. The intensity is typically a factor of two higher if the beam is pointed onto the area between the grooves.

For the control sample in Fig. 2(a) a very weak periodic pattern of emission is observed between the grooves, which is symmetric around the center of the plateau. The amplitude of the oscillations decreases away from the grooves toward the center, and only a diffuse pattern is observed. The period

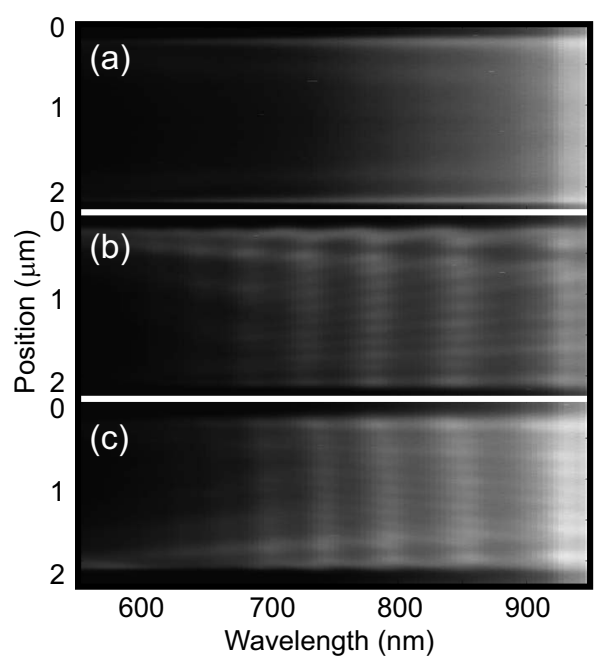

FIG. 2. Cathodoluminescence intensity as a function of position and wavelength for line scans across three $\mathrm{Ag} / \mathrm{SiO}_{2} / \mathrm{Ag} \mathrm{MIM}$ Fabry-Perot resonators with $2000 \mathrm{~nm}$ groove separation. Brighter colors indicate stronger CL emission. (a) Control sample without $\mathrm{SiO}_{2}$ layer. (b) MIM structure with a $50 \mathrm{~nm} \mathrm{SiO}_{2}$ layer. (c) MIM structure with a $30 \mathrm{~nm} \mathrm{SiO}_{2}$ layer.

of the oscillations increases almost linearly with increasing wavelength and is approximately half the free-space wavelength.

The MIM sample with a $50 \mathrm{~nm} \mathrm{SiO}_{2}$ layer [Fig. 2(b)] shows a more intense oscillatory pattern. Seven bands of higher emission are observed for wavelengths of 610, 640, $680,730,780,850$, and $920 \mathrm{~nm}$. The emission from the center of the resonator at these wavelengths shows oscillations with a period that is much shorter than the detected light wavelength. The amplitude and visibility of the oscillation fringes in each wavelength band strongly decrease for shorter wavelengths. Near the edges, a larger-period oscillation is superimposed comparable to the one in Fig. 2(a).

The MIM sample with a $30 \mathrm{~nm} \mathrm{SiO}_{2}$ layer [Fig. 2(c)] shows eight bands of high emission at 600, 630, 660, 695, $740,790,850$, and $920 \mathrm{~nm}$. The period of the oscillations for these wavelength bands is slightly shorter than for the $50 \mathrm{~nm}$ structure.

The observed CL emission of the measured samples stems from three sources that have to be taken into account: transition radiation (TR), radiation from SPPs propagating at the $\mathrm{Ag} / \mathrm{Cr}$ interface, and radiation from MIM plasmons.

The TR contribution was determined by measuring the CL emission at a sample position where no structures are present that could scatter plasmons. The TR spectrum is very similar for all three samples, indicating that it is mainly determined by the uppermost layer.

First, we analyze the measurements of the control sample. The oscillations close to the grooves are attributed to interference of scattered SPPs with TR. ${ }^{9}$ The fact that only one oscillation is observed (rather than the extensive pattern in Ref. 9) is due to the fact that the propagation length of the surface SPPs is less than $1 \mu \mathrm{m}$.

The MIM sample with the $50 \mathrm{~nm} \mathrm{SiO}_{2}$ layer also shows these oscillations near the edges, but in addition clear oscillations with a period much shorter than half the free-space 
wavelength. We attribute these to Fabry-Perot modes of MIM plasmons that are reflected between the grooves.

To analyze the data, we have determined the period of the observed oscillations by fitting the CL intensity as a function of position $x$ for each band with a function $\propto \sin ^{2}\left(k_{\mathrm{MIM}} x\right)$, with $k_{\mathrm{MIM}}=2 \pi / \lambda_{\mathrm{MIM}}$ the MIM plasmon wave vector. We find plasmon wavelengths of $330,350,370$, and $430 \mathrm{~nm}$ for the light wavelength bands at 730, 780, 850, and $920 \mathrm{~nm}$, respectively. For the bands at shorter wavelengths, the oscillations are damped out too strongly to allow us to fit their period. However, we can still derive the MIM plasmon wavelength for these bands from their mode number.

Each band of high emission in the CL scans corresponds to a cavity mode with a successive mode number $n$ given by $2 d k_{\mathrm{MIM}}+\phi=2 n \pi$ where $d$ is the resonator width and $\phi$ is the phase change upon reflection (we assume $\phi=0$ ). For the above identified bands, we find mode numbers of $n=9$ (920 $\mathrm{nm}), n=10(850 \mathrm{~nm}), n=11(780 \mathrm{~nm})$, and $n=12(730 \mathrm{~nm})$. Therefore, the subsequent lower-wavelength bands should have mode numbers $n=13(680 \mathrm{~nm}), n=14(640 \mathrm{~nm})$, and $n=15(610 \mathrm{~nm})$. Given the resonator width, we can then derive the corresponding MIM plasmons wavelength as 305, 285, and $266 \mathrm{~nm}$.

The MIM sample with a $30 \mathrm{~nm} \mathrm{SiO}_{2}$ layer shows eight bands of high emission corresponding to eight modes of the Fabry-Perot resonator. The bands are more closely spaced, corresponding to a smaller free-spectral range, which is in agreement with the shorter plasmon wavelengths expected for this sample. The stronger damping of the plasmons in this sample compared to the $50 \mathrm{~nm} \mathrm{SiO}_{2}$ sample leads to a smaller oscillation amplitude. Due to the stronger damping only the oscillation period of the three longest-wavelength bands can be fitted for this sample. We find plasmon (free space) wavelengths of $320 \mathrm{~nm}(780 \mathrm{~nm}), 340 \mathrm{~nm}(850 \mathrm{~nm})$, and $390 \mathrm{~nm}(920 \mathrm{~nm})$ corresponding to mode numbers of $n=12, n=11$, and $n=10$, respectively. From the mode number the plasmon wavelengths for the other bands are derived to be $227 \mathrm{~nm}(600 \mathrm{~nm}), 240 \mathrm{~nm}(630 \mathrm{~nm}), 255 \mathrm{~nm}(660 \mathrm{~nm})$, $272 \mathrm{~nm}(695 \mathrm{~nm})$, and $280 \mathrm{~nm}(740 \mathrm{~nm})$.

For the sample with a $10 \mathrm{~nm} \mathrm{SiO}_{2}$ layer, bright bands are observed at 645, 663, 680, 704, 734, 765, 805, 846, and 892 $\mathrm{nm}$. Since the wavelength of the MIM plasmons is too short to resolve oscillations and fit their period, we have used the analytical dispersion relation to calculate the mode number $n=17$ for the band at $892 \mathrm{~nm}$. From the subsequent mode numbers $n=18-25$ we derived the wave vectors for the higher-energy bands.

Figure 3 shows the MIM mode dispersion relation for the 10, 30, and $50 \mathrm{~nm}$ thick $\mathrm{SiO}_{2}$ layers, with data points as derived above. The analytical dispersion relation for the symmetric MIM mode is also plotted, calculated from the optical constants for the layer stack measured using spectroscopic ellipsometry. The experimental data agree remarkably well with the calculation and lie far to the right of the light line in $\mathrm{SiO}_{2}$, thus demonstrating the strongly dispersive character of these MIM modes. The good agreement between experiment and the calculated dispersion relation for symmetric MIM plasmons, confirms our assumption that the observed plasmon modes are of symmetric character.

To illustrate the excitation mechanism in our experiment,

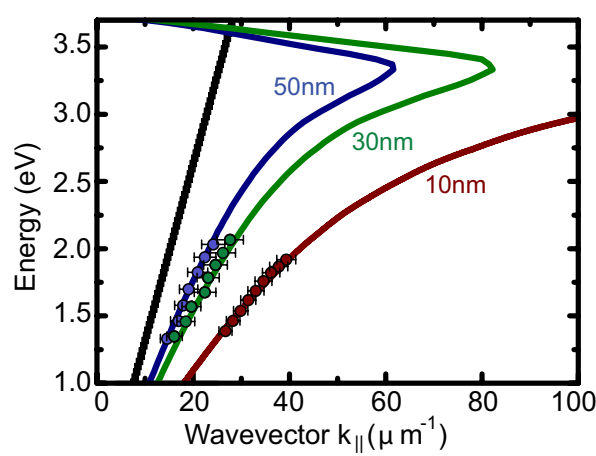

FIG. 3. (Color online) Dispersion relation for symmetric $\mathrm{Ag} / \mathrm{SiO}_{2} / \mathrm{Ag} \mathrm{MIM}$ plasmons with a $10 \mathrm{~nm}$ (red), $30 \mathrm{~nm}$ (green), and $50 \mathrm{~nm} \mathrm{SiO}$ layer (blue). The dots are derived from the cathodoluminescence line scans of Fig. 2. The solid lines are analytical calculations based on measured dielectric constants. The light line in $\mathrm{SiO}_{2}$ is drawn in black.

we have investigated the excitation probability of MIM plasmons by an electron beam. For the studied MIM cavities the incident electron crosses the boundary between the metal and $\mathrm{SiO}_{2}$ twice, generating excitations at both metal-dielectric interfaces. Due to the finite velocity of the electrons, the plasmon fields excited at the two interfaces will have a phase difference $\Delta \phi$ depending on the thickness $d$ of the $\mathrm{SiO}_{2}$ core and the electron velocity $v$ :

$$
\Delta \phi=2 \pi \frac{d}{v} \frac{c}{\lambda}+\phi_{0},
$$

where $\lambda$ is the free-space wavelength. The phase factor $\phi_{0}$ accounts for the phase difference between the plasmon fields generated at the electron transition from metal to dielectric and vice versa; for the MIM structure $\phi_{0}=\pi$.

Figure 4(a) shows the calculated phase difference as a function of electron velocity at a free-space wavelength of $800 \mathrm{~nm}$ for three different $\mathrm{SiO}_{2}$ layer thicknesses of 30, 50, and $100 \mathrm{~nm}$. The phase difference increases inversely with decreasing electron velocity and linearly with layer thickness. The inset of Fig. 4 shows the schematic magnetic field profiles for the symmetric and antisymmetric MIM modes.

The phase difference leads to constructive or destructive interference between excitations on the two interfaces. The experimentally observed MIM modes are of symmetric character and have the highest-field intensity in the dielectric core as shown in the inset of Fig. 4(a). Therefore, we expect a decrease in emission probability for symmetric MIM plasmon at phase difference of $\Delta \phi=(2 n+1) \pi$, with integer $n$ [indicated by dashed lines in Fig. 4(a)].

At the marked positions, as well as in the limit of large $v$ and small dielectric thickness a plasmon mode with antisymmetric magnetic field distribution (see inset of Fig. 4) will be preferentially excited. We attribute absence of these modes in our measurements to the fact that their propagation length is extremely short. ${ }^{3}$

The emission probability for the symmetric plasmon mode in an MIM cavity was calculated as described in Ref. 11. The results are shown in Fig. 4(b) as a function of electron velocity for $\mathrm{SiO}_{2}$ layer thicknesses of 30, 50, and 100 


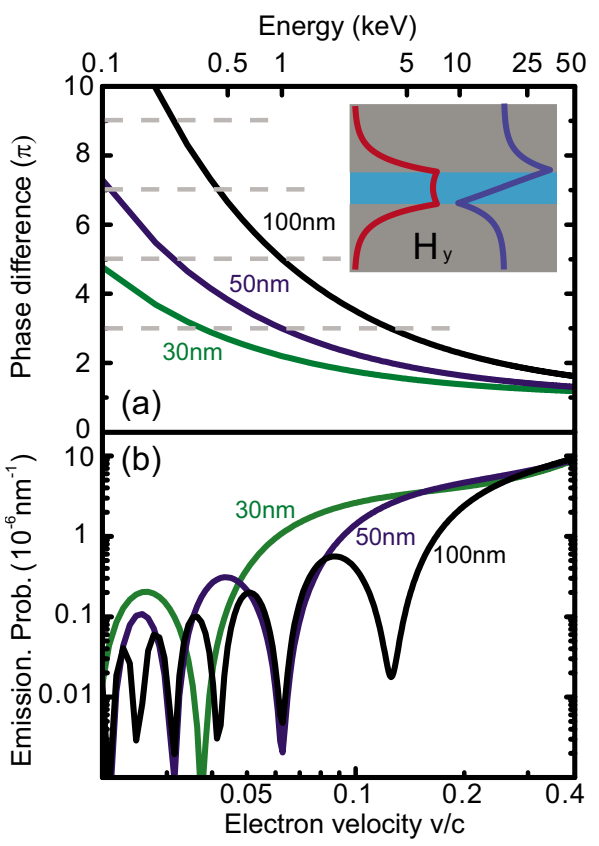

FIG. 4. (Color online) (a) Phase difference for plasmon excitation (free-space wavelength $800 \mathrm{~nm}$ ) by an electron passing through a MIM structure as a function of electron velocity. The phase difference was calculated for an $\mathrm{SiO}_{2}$ thickness of $30 \mathrm{~nm}$ (green), 50 $\mathrm{nm}$ (blue), and $100 \mathrm{~nm}$ (black line). The gray horizontal dashed lines denote phase differences of $(2 n+1) \pi$ with integer $n$. Inset: Schematic of the magnetic field profile for the symmetric (red) and antisymmetric (blue) MIM mode. (b) Plasmon emission probability as a function of electron velocity for the symmetric MIM mode.

nm. We observe a decrease in the excitation rate with decreasing electron velocity, in agreement with higher localization of the external field produced by the electron. Superimposed on this decrease, we observe dips in the emission probability for electron velocities at which the phase difference equals an odd number times $\pi$. Moreover, the number of dips increases with layer thickness in agreement with Eq. (1).

These calculations indicate that it is possible to selectively excite a plasmon mode with desired symmetry by varying the beam energy. For the studied samples, the dips of highest energy with reduced emission of symmetric MIM modes are found at very low-beam energies: $0.37 \mathrm{keV}$ for the $30 \mathrm{~nm}$ $\mathrm{SiO}_{2}$ layer (green curves) and $1.01 \mathrm{keV}$ for the $50 \mathrm{~nm} \mathrm{SiO}$ layer (blue curves). For these energies the inelastic mean free path in Ag is very short ( 9 and $15 \mathrm{~nm}$, respectively ${ }^{12}$ ) and the electron transmission through the upper Ag layer is thus extremely low. For a $\mathrm{SiO}_{2}$ layer thickness of $100 \mathrm{~nm}$, a dip of reduced emission is found for an energy of $4 \mathrm{keV}$. In this case the inelastic mean free path for the electron is $43 \mathrm{~nm}$.

Due to the constraint of reduced electron transmission through the top Ag layer at low energies, it is difficult to experimentally demonstrate selective excitation of certain plasmon symmetry for normal-incidence electrons. However by tilting the sample with respect to the electron beam the path length $d$ through the $\mathrm{SiO}_{2}$ layer can be increased, so that selective excitation can be driven through the effective $d$ rather than $v$ in a modified version of Eq. (1). The fact that the excitation is generated at different lateral positions leads to an additional phase difference. The tilt required to achieve a minimum in symmetric mode excitation such as in Fig. 4(b) for electrons incident on an MIM structure with a 100 $\mathrm{nm} \mathrm{SiO}$ layer at $10 \mathrm{keV}$ is $35^{\circ}$ for a wavelength of $800 \mathrm{~nm}$. For $30 \mathrm{keV}$ electrons the sample has to be tilted to $51^{\circ}$.

We have shown that cathodoluminescence imaging can be used to excite and map plasmonic modes in $\mathrm{Ag} / \mathrm{SiO}_{2} / \mathrm{Ag}$ MIM structures. The generated MIM plasmons were confined in Fabry-Perot resonators. From the spatially resolved mode profile of the Fabry-Perot resonators the dispersion relation of the MIM plasmons was determined, and found to be in good agreement with theory. Additionally, we have shown that the excitation probability of resonant MIM plasmons depends strongly on the electron energy due to phase retardation effects resulting from the finite electron velocity.

This work is part of the research program of FOM, which is financially supported by NWO. W.C. and F.J.G.A. acknowledge support from the Spanish MEC (Contract No. MAT2007-66050).

\footnotetext{
*kuttge@amolf.nl

${ }^{1}$ H. T. Miyazaki and Y. Kurokawa, Phys. Rev. Lett. 96, 097401 (2006).

${ }^{2}$ E. N. Economou, Phys. Rev. 182, 539 (1969).

${ }^{3}$ J. A. Dionne, L. A. Sweatlock, H. A. Atwater, and A. Polman, Phys. Rev. B 73, 035407 (2006).

${ }^{4}$ L. Chen, J. Shakya, and M. Lipson, Opt. Lett. 31, 2133 (2006).

${ }^{5}$ E. Verhagen, J. A. Dionne, L. Kuipers, H. A. Atwater, and A. Polman, Nano Lett. 8, 2925 (2008).

${ }^{6}$ H. J. Lezec, J. A. Dionne, and H. A. Atwater, Science 316, 430 (2007).
}

${ }^{7}$ J. A. Dionne, E. Verhagen, A. Polman, and H. A. Atwater, Opt. Express 16, 19001 (2008).

${ }^{8}$ Z. Zhu and T. G. Brown, Opt. Express 10, 853 (2002)

${ }^{9}$ M. Kuttge, E. J. R. Vesseur, A. F. Koenderink, H. J. Lezec, H. A. Atwater, F. J. Garcia de Abajo, and A. Polman, Phys. Rev. B 79, 113405 (2009)

${ }^{10}$ D. Drouin, A. Réal Couture, D. Joly, X. Tastet, V. Aimez, and R. Gauvin, Scanning 29, 92 (2007).

${ }^{11}$ W. Cai, R. Sainidou, J. Xu, A. Polman, and F. J. García de Abajo, Nano Lett. 9, 1176 (2009).

${ }^{12}$ D. R. Penn, Phys. Rev. B 35, 482 (1987). 